\title{
Current understanding of the administration of mesenchymal stem cells in acute kidney injury to chronic kidney disease transition: a review with a focus on preclinical models
}

\author{
Lingfei Zhao ${ }^{1,2}$, Fei Han ${ }^{1,2}$, Junni Wang ${ }^{1,2}$ and Jianghua Chen ${ }^{1,2^{*}}$ (D)
}

\begin{abstract}
Incomplete recovery from acute kidney injury (AKI) can result in long-term functional deficits and has been recognized as a major contributor to chronic kidney disease (CKD), which is termed the AKI-CKD transition. Currently, an effective intervention for this disorder is still lacking. Principally, therapeutic strategies targeting the AKI-CKD transition can be divided into those reducing the severity of AKI or promoting the regenerative process towards beneficially adaptive repair pathways. Considering the fact that mesenchymal stem cells (MSCs) have the potential to address both aspects, therapeutic regimens based on MSCs have a promising future. In light of this information, we focus on the currently available evidence associated with MSC therapy involved in the treatment of the AKI-CKD transition and the underlying mechanisms. All of these discussions will contribute to the establishment of a reliable therapeutic strategy for patients with this problem, who can be easily ignored by physicians, and will lead to a better clinical outcome for them.
\end{abstract}

Keywords: Mesenchymal stem cells, AKI-CKD transition

\section{Background}

Despite advances in modern medicine, the incidence, prevalence, and mortality of chronic kidney disease (CKD) have increased in the last 10 years [1]. CKD is characterized as a continuously advancing loss of renal function and irreversible accumulation of extracellular matrix. According to a US renal data system annual report, approximately $13.6 \%$ of people in the USA are suffering from CKD [2]. The high morbidity and poor prognosis place a huge burden on public health systems, and it is estimated that over 49 billion dollars are spent annually for treating CKD patients [3].

One major reason for the development of CKD is acute kidney injury (AKI). Contrary to the traditional view that

\footnotetext{
* Correspondence: zjukidney@zju.edu.cn

${ }^{1}$ Kidney Disease Center, the First Affiliated Hospital, College of Medicine,

Zhejiang University, Hangzhou, Zhejiang Province, People's Republic of China

${ }^{2}$ Key Laboratory of Kidney Disease Prevention and Control Technology,

Zhejiang Province, Institute of Nephrology, Zhejiang University, Hangzhou,

Zhejiang Province, People's Republic of China
}

AKI and CKD are considered as two distinct syndromes, numerous animal studies and epidemiological evidence in recent years provide a strong possibility that AKI and CKD share an interconnected pathophysiological process [4]. Unlike other powerfully regenerative organs, such as the heart or liver, injured kidneys that suffer from AKI usually do not have the capacity to completely repair themselves [5]. Maladaptive kidneys will undergo fibroblast activation and the deposition of the extracellular matrix, which may contribute to a vicious cycle of fibrogenesis and nephron loss and ultimately induce the development of CKD [6, 7]. For this reason, it is easy to understand the fact that AKI patients who survive the acute phase will bear a 13-fold higher risk of developing CKD in their lifetime. For those patients with AKI at the RIFLE failure stage, the risk of progression to CKD is up to 41 times higher [8]. The phenomenon of progression to CKD after AKI is termed the AKI-CKD transition [9].

(c) The Author(s). 2019 Open Access This article is distributed under the terms of the Creative Commons Attribution 4.0 International License (http://creativecommons.org/licenses/by/4.0/), which permits unrestricted use, distribution, and 
During the last decade, multiple therapeutic strategies have been explored for targeting the AKI-CKD transition; however, few faithful pharmacologic agents have been proven to be able to effectively block this transition. Interventions with hypoxia-inducible factor (HIF), vascular endothelial growth factor (VEGF), or nuclear factor erythroid-2-related factor 2 (Nrf2) present promising outcomes in some studies, but contradictions still exist [10-13]. Currently, the strongest evidence for the management of disease progression still comes from the way in which we manage established CKD, namely, renin-angiotensin system inhibitor (RASI). However, this strategy provides no help for renal regeneration $[14,15]$. Exploring novel interventions that have the capacity to promote renal regeneration and halt AKI-CKD transition is an urgent need.

MSCs are a type of stem cell which are characterized by their robust capacities of self-renewal, regeneration, proliferation, and three-lineage differentiation [16]. Multiple animal injury models, including the lung, liver, and Alport's syndrome, have confirmed that MSCs are able to reverse organ fibrosis [17-19]. Compared with pharmacologic interventions, which target only one single aspect of the highly complex pathophysiological process during the AKI-CKD transition, MSCs may have the advantage of presenting multiple regenerative effects for organ protection [20]. It is thought that MSCs possess intrinsic properties of immunomodulation, proangiogenesis, anti-inflammation, anti-apoptosis, and antioxidation, which are greatly beneficial for limiting acute injury and reversing chronic progression [21-23].

However, to date, most studies in the field of regenerative medicine are still confined to only evaluating the therapeutic effects of MSCs on either AKI or CKD [24, 25]. Few research studies have paid attention to issues regarding the AKI-CKD transition. It seems that a knowledge gap still exists regarding the connection between AKI and CKD. Given that this concept was just proposed in the last decade, it is also comprehensible to understand the situation [26]. Fortunately, the phenomenon of chronic sequelae of AKI is receiving more and more attention in the last few years based on the fact that there has been a dramatic increase in research. Regenerative medicine physicians are also wondering how to regard the role of MSCs in this area. Due to these conditions, we collected associated evidence about the administration of MSCs in AKI-CKD transition. This is the first review article related to this issue thus far. By summarizing available articles and discussing the underlying mechanisms in this review, we intend to provide an integrated and up-to-date view of the application of MSCs in the AKI-CKD transition and call for a focus on issues related to long-term ramifications after AKI, with the aim of improving prognosis in AKI patients and delaying the progression to CKD.

\section{Maladaptive repair after $\mathrm{AKI}$ and the mechanisms involved in the transition}

Several years ago, it was thought that the repair process after AKI might be fully adaptive and that surviving patients who recovered renal function would not suffer from long-term consequences [27]. Lineage-tracing studies indicated that tubular epithelial cells (TECs) were thought to be the main source of cell regeneration after injury $[28,29]$. During the repair process, surviving TECs underwent dedifferentiation, migration along the basement membrane, proliferation, and finally redifferentiation. After these orchestrated reparative processes, damaged TECs were replaced with functional new ones, and a refreshed steady state was established [30, 31]. However, due to the importance of TECs, a maladaptive response in TECs is also thought to occupy a central position in the transition from AKI to CKD, regardless of the diverse injuries in the blood vessels, glomeruli, and inflammatory/immune cells [26]. An episode of injury will lead to capillary rarefaction, mitochondrial injury and metabolic disorder, epigenetic alterations, persistent inflammation, profibrogenic signal production, and fibroblast/myofibroblast expansion, which will ultimately cause the development of CKD (Fig. 1) [32-38]. In the following section, we would like to discuss these chronic sequelae after AKI and the relevant biochemical pathways related to its progression.

\section{Capillary rarefaction}

Unlike TECs, vascular endothelial cells possess less regenerative potential [39]. Accompanied by low expression levels of VEGF after AKI, one AKI strike may result in disastrous consequences to the renal tissue microvasculature $[38,40]$. It was reported that a massive reduction of capillary density occurred after inducing AKI in a rat model [41]. Capillary rarefaction could exacerbate tissue hypoxia, both of which no doubt play critical roles in promoting interstitial fibrosis and the progression to CKD [42, 43]. Based on these facts, Kapitsinou et al. applied pharmacologic HIF agonists to prevent the development of fibrosis after AKI and showed positive results, which in turn verified this hypothesis [10].

\section{Mitochondrial injury and metabolic disorder}

Capillary rarefaction and hypoxia initiate mitochondrial injury and metabolic disorder, especially in proximal TECs and medullary thick ascending limb TECs, which largely rely on oxidative metabolism to provide the vast amount of adenosine triphosphate (ATP) they require for tubular reabsorption [44]. Pathologically, injured mitochondria present with reduced numbers, fragmented shape, and formation of autophagosomes [45, 46]. Functionally, cells switched from oxidative phosphorylation to glycolysis, inducing fat accumulation, together 


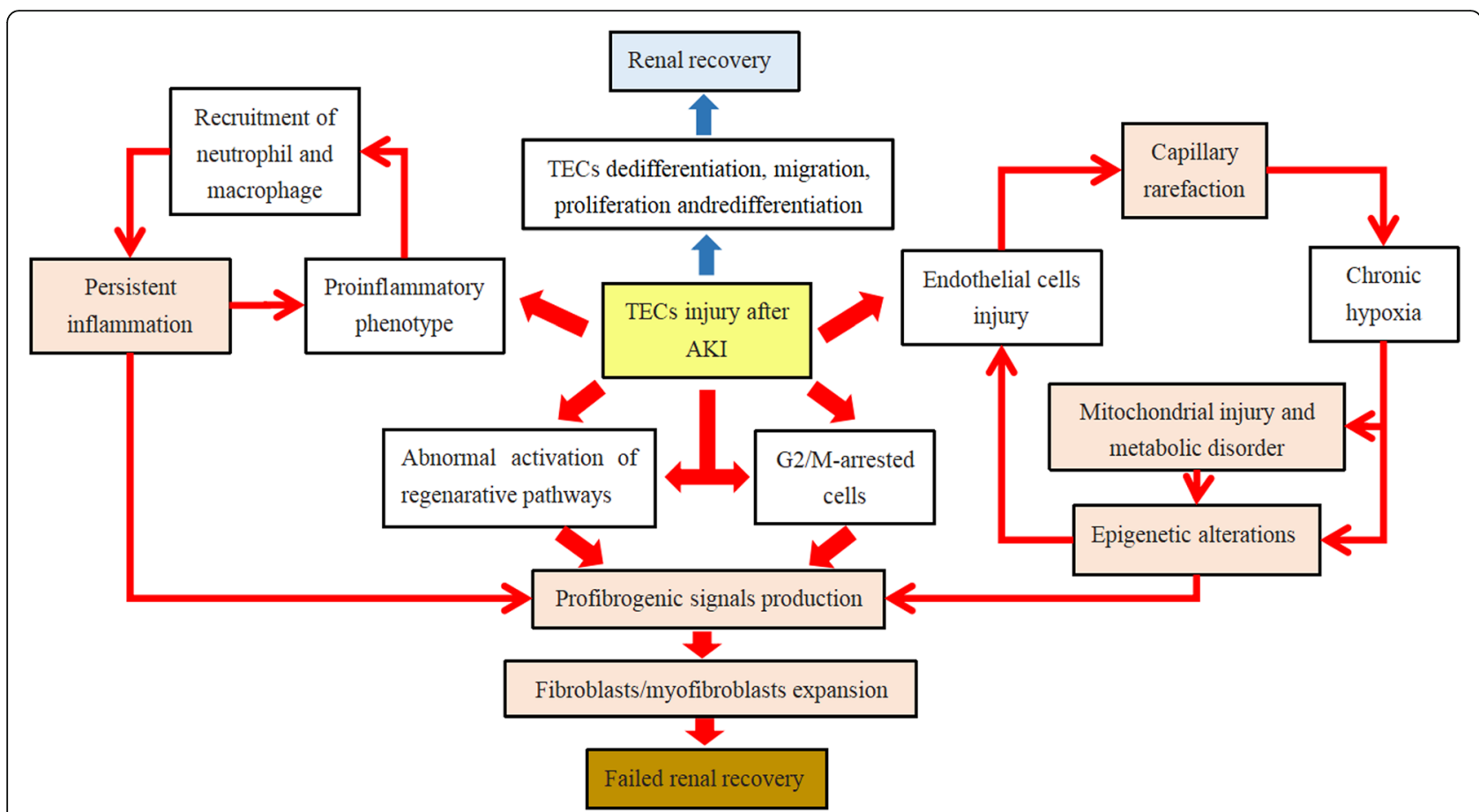

Fig. 1 Adaptive and maladaptive response in TECs after AKI. Under certain circumstances, injured TECs are able to dedifferentiate, migrate, proliferate, and finally redifferentiate into normal TECs, promoting renal recovery (blue arrows). While AKI is severe or episodes are frequent, TECs may lose their regenerative capacity and undergo maladaptive repair. The major pathophysiological alterations during this process include capillary rarefaction, mitochondrial injury and metabolic disorder, epigenetic alterations, persistent inflammation, and other effects. These changes may interact with each other and can ultimately result in profibrogenic signal production, fibroblast/myofibroblast expansion, and failed renal recovery (red arrows)

with the downregulation of multiple electron transport chain and ATP synthesis genes [45, 47]. Moreover, the release of dangerous molecules by mitochondria, such as reactive oxygen species (ROS), would be activated if long-term injury existed, which sustained chronic inflammation and delayed renal recovery [48, 49]. All of these alternations in mitochondria contribute to mediating glomerulosclerosis and interstitial fibrosis after AKI.

\section{Epigenetic alterations}

In addition to the metabolic and structural changes in the mitochondria, disturbances in epigenetic modifications can also be activated after AKI. Considering that histone deacetylase is a key regulator of the expression of fibrosis-related genes, Novitskaya et al. and Cianciolo et al. tried to determine if aberrant histone deacetylase contributed to AKI-CKD transition in two different AKI-CKD transition models. Acceleration of AKI recovery and prevention of post-injury fibrosis were observed after the inhibition of histone deacetylase [50,51]. Apart from histone deacetylase, there is an extensive crosstalk between histone modification and DNA methylation [52]. A subsequent study by Tampe et al. showed that the administration of low-dose hydralazine efficiently catalyzed RASAL1 demethylation and inhibited tubulointerstitial fibrosis [53]. The results from these studies confirmed the crucial roles that epigenetic alterations played in AKI-CKD progression.

\section{Persistent inflammation}

AKI is no doubt closely linked with inflammation. Tubulointerstitial inflammation can be observed in almost all types of AKI in the early stage, whereas persistent inflammation inevitably results in the progression of fibrosis. The interaction between tubulointerstitial inflammation and TECs is a key determinant in the development of persistent inflammation. Multiple identical pattern recognition receptors, including Toll-like receptors (TLRs), Nod-like receptors, and NLRP3 inflammasomes, were found to be widely expressed in TECs [54]. During the early stage of AKI, tubulointerstitial inflammation triggered by pathologic factors, such as ischemia, toxins, or proteinuria, could activate the TLR2-MyD88-NF-kB pathway and upregulate NLRP3 inflammasomes $[55,56]$. These alternations in TECs transformed them into a proinflammatory phenotype and elicited the secretion of various proinflammatory mediators, including tumor necrosis factor (TNF)- $\alpha$, IL-6, IL-1 $\beta$, IL15, IL-16, and VEGF, among others [57-61]. The release of proinflammatory cytokines recruited neutrophils and infiltrating macrophages, polarized macrophages/dendritic cells, facilitated the immune response, and led to persistent AKI and subsequent CKD $[62,63]$. These results support a role 
for persistent inflammation in the pathogenesis of the AKICKD transition.

\section{Profibrogenic signal production}

Multiple signal pathways can be activated during the recovery phase of AKI due to the requirement of cell regeneration, but all of these pathways should be ceased following renal recovery. Abnormal activation of these pathways can induce pathological outcomes. Examples include Wnt/b-catenin and miR-21/phosphatase and tensin homolog (PTEN) signaling. The activation of Wnt-4/b-catenin in the acute phase of injury allows injured cells to re-enter the cell cycle and regulates cell proliferation, which is helpful in renal recovery $[64,65]$. However, sustained activation of Wnt signaling is accompanied by the development of interstitial myofibroblast activation and excessive extracellular matrix deposition [66]. A similar situation was also observed in the case of miR-21/PTEN signaling [67, 68]. Disturbance in the regulation of these pathways transformed regenerative signals into profibrogenic signals.

Apart from the abovementioned mechanism, the expansion of profibrogenic signals can also be driven by G2/M-arrested cells. Unlike adaptive repair, which induced surviving TECs to undergo continuous four-phase mitosis for proliferation (G0, G1, S, G2 and M), particularly stressful conditions during AKI may lead some TECs to remain arrested in the G2/M phase. These TECs are active in the production of profibrogenic signals, such as transforming growth factor- $\beta$ (TGF- $\beta$ ), cjun NH2-terminal kinase (JNK), and epidermal growth factor receptor (EGFR), which provide a pathophysiological link between AKI and CKD [35, 69, 70].

\section{Fibroblast/myofibroblast expansion}

Fibroblast/myofibroblast expansion is the final procedure for the development of fibrosis. After stimulation by the abovementioned pathophysiological alternations, including capillary rarefaction, mitochondrial injury and metabolic disorder, epigenetic alterations, persistent inflammation, and profibrogenic factor production, interstitial precursor cells finally differentiate into fibroblasts/ myofibroblasts. Although the origin of fibroblasts/myofibroblasts is still under debate, pericytes seem to be the major source. Normally, pericytes are cells of mesenchymal origin with branching processes that form junctions between the capillaries and tubules [71]. Under stressful conditions such as AKI, harmful signals, such as platelet-derived growth factor receptor- $\beta$ (PDGFR- $\beta$ ), VEGF receptor 2, or $\alpha$-engagement, can drive pericytes to detach from the capillaries. Detached pericytes spread, migrate, and transform into myofibroblasts [32, 72]. In addition to pericytes, some research studies have also regarded TECs, endothelial cells, or bone marrow cells as other sources of fibroblasts/myofibroblasts [7, 38]. The continuous expansion of fibroblasts/myofibroblasts, with excessive production of collagen, widens the normal tubulointerstitial space and injured endothelium regresses, causing kidney fibrosis [73].

\section{MSCs are a promising candidate for the management of the AKI-CKD transition}

Above, we discussed the pathophysiological alterations during the AKI-CKD transition. It should be noted that the abovementioned pathophysiological changes are not merely separated processes; rather, they interact with each other in a complicated network. For example, as described by Liu et al. in their article, TECs under injury can undergo a proinflammatory phenotype change and drive interstitial inflammation. In turn, activated infiltrating immune cells can also induce TEC necrosis and exaggerated renal injury [74]. Similarly, Venkatachalam et al. also demonstrated that although renal epithelial dysfunction with ensuing paracrine activity is the initial issue in this scheme, subsequent interactions between different pathophysiological processes, such as inflammation, fibroblasts, and capillary rarefaction, can form a self-reinforcing cycle feedback [75]. These lines of evidence make it difficult to assign their roles as "chicken and egg" in many cases. Additionally, for this reason, it is not hard to understand the limited therapeutic effects of drug interventions in the AKI-CKD transition because one single drug approach commonly only targets one single pathway.

The advantage of MSC-based therapy over drug interventions in attenuating the AKI-CKD transition is due to its multipotent regenerative properties both in vitro and in vivo, which cover most of its highly complex pathophysiological processes. Although only scarce evidence has demonstrated that MSCs can replace injured tissues in vivo, the capacity to differentiate into all mesodermal lineage cells was well verified in vitro, and this potential was full of interest and imagination [76]. The most widely accepted proregenerative capability of MSCs relied on its paracrine/endocrine capacity. Accumulating evidence has emphasized that MSCs are active in the release of a number of cytokines/growth factors, such as hepatocyte growth factor (HGF), fibroblast growth factor (FGF), VEGF, TGF- $\beta$, and insulin-like growth factor type 1 (IGF-1) [77]. These biological molecules can exert anti-apoptotic, anti-oxidative, and pro-angiogenic effects, promoting local cell recovery [78-80]. Recent findings have also suggested that MSCs can alleviate renal injury by secreting extracellular vesicles (EVs), which is regarded as another special form of paracrine/endocrine response. EVs are important cell-to-cell interaction mediators and are rich in a broad variety of biologically active molecules, including lipids, proteins, and nucleic 
acids (e.g., mRNAs, miRNAs, and lncRNAs) [81]. After internalization, the biologically active molecules inside EVs can be transmitted into the target cells, changing their phenotype and exerting biological effects [82]. Except for the secretion of bioactive substances, MSCs can also act as an ideal vehicle for organelle delivery. For example, by transferring healthy mitochondria, defective native ones in injured renal cells can be substituted, and aerobic respiration can be revitalized [44]. Based on these regenerative properties, it is not surprising that MSCs are regarded as a promising candidate in the AKICKD transition. In the following section, we will talk about the application of MSCs in different AKI-CKD transition models and the mechanisms underlying their beneficial effects (Table 1). Most of the currently available data has come from ischemia-reperfusion (I/R) injury-induced AKI-CKD transition models.

\section{Application of MSCs in the unilateral I/R injury-induced AKI-CKD transition model}

The unilateral $I / R$ injury model is a widely used model in the study of the AKI-CKD transition. This model is induced by clamping the pedicle of only one kidney. Fibrosis of the renal tissues followed by the ischemic strike can be observed in this model, making it a reliable strategy to study the AKI-CKD transition [83].

Zhu et al. transferred adipose-derived MSCs (A-MSCs) into a mouse model of unilateral I/R injury-induced AKICKD transition. The administration of A-MSCs significantly alleviated the fibrosis and atrophy of renal tissues 28 days after the severe event of AKI. Molecularly, $\alpha$-smooth muscle actin $(\alpha-$ SMA $)$ and collagen-I were reduced at both the
mRNA and protein levels, suggesting the attenuation of fibrosis. To further explore the mechanism underlying the beneficial effects of A-MSCs on the AKI-CKD transition, they assessed the expression of TGF- $\beta$, an important profibrotic factor in renal tissues. As expected, a reduced expression of TGF- $\beta 1$ and a decreased level of phosphorylation Smad3 were observed [84]. Similar outcomes were obtained by Masoud et al. In their study, animals injected with MSCs presented improved renal fibrosis after clamping the left renal pedicle for $45 \mathrm{~min}$ [85]. MSCs are the main component of bone marrow mononuclear cells (BMMCs). BMMC therapy after unilateral I/R injury could significantly halt its progression to chronic fibrosis. After 6 weeks of transplantation, although the serum creatinine level was not changed, fewer fibrosis areas and less proteinuria together with larger renal areas in the injured kidneys were observed in animals that received BMMC therapy when compared with untreated animals. At the molecular level, pro-fibrotic molecules, such as $\alpha$-SMA, vimentin, and fibroblast-specific protein-1 (FSP-1), presented similar patterns in the kidneys of BMMC-treated animals, suggesting a protective role of BMMCs in attenuating the AKI-CKD transition [86].

\section{Application of MSCs in the unilateral I/R injury with contralateral nephrectomy injury-induced AKI-CKD transition model}

All of the abovementioned studies lack an outcome in renal function. This is due to a major disadvantage of the unilateral $\mathrm{I} / \mathrm{R}$ injury-induced AKI-CKD transition model. With a functional and compensatory contralateral kidney, it is always difficult to monitor the renal functional decline. One way to partially overcome this

Table 1 Evidence suggesting the beneficial effects of MSCs on the AKI-CKD transition

\begin{tabular}{|c|c|c|c|c|}
\hline References & Year & Animal & Model & Renal outcomes \\
\hline Zhu et al. [84] & 2017 & Mice & Unilateral I/R injury & $\downarrow$ Fibrosis; $\uparrow$ kidney weight and size; $\downarrow$ a-SMA and collagen-l; $\downarrow$ TGF- $\beta 1 /$ Smad3 \\
\hline Masoud et al. [85] & 2012 & Rats & Unilateral I/R injury & $\downarrow$ Fibrosis \\
\hline Semedo et al. [86] & 2010 & Mice & Unilateral I/R injury & $\downarrow$ Fibrosis; $\downarrow$ proteinuria; $\uparrow$ renal areas; $\downarrow$ a-SMA, vimentin, and FSP-1 \\
\hline Gatti et al. [88] & 2011 & Rats & $\begin{array}{l}\text { Unilateral I/R injury with } \\
\text { contralateral nephrectomy }\end{array}$ & $\uparrow$ Renal function; $\downarrow$ fibrosis; $\downarrow$ proteinuria \\
\hline Du et al. [90] & 2012 & Rats & $\begin{array}{l}\text { Unilateral I/R injury with } \\
\text { contralateral nephrectomy }\end{array}$ & $\uparrow$ Renal function; $\downarrow$ fibrosis \\
\hline Zou et al. [89] & 2014 & Rats & $\begin{array}{l}\text { Unilateral I/R injury with } \\
\text { contralateral nephrectomy }\end{array}$ & $\uparrow$ Renal function; $\downarrow$ fibrosis; $\downarrow$ a-SMA \\
\hline Alfarano et al. [91] & 2012 & Rats & $\begin{array}{l}\text { Unilateral I/R injury with } \\
\text { contralateral nephrectomy }\end{array}$ & $\uparrow$ Renal function; $\downarrow$ fibrosis and tubular dilation; $\downarrow$ a-SMA and MMP2 \\
\hline Zhou et al. [93] & 2016 & Rats & $\begin{array}{l}\text { Unilateral I/R injury with } \\
\text { contralateral nephrectomy }\end{array}$ & $\uparrow$ Renal function; $\downarrow$ fibrosis; $\downarrow$ a-SMA; $\downarrow$ microvascular rarefaction \\
\hline Tögel et al. [94] & 2009 & Rats & Bilateral I/R injury & $\uparrow$ Renal function; $\downarrow$ fibrosis; $\downarrow$ PAl- 1 and TGF- $\beta$ \\
\hline Du et al. [96] & 2013 & Rats & Bilateral I/R injury & $\downarrow$ Fibrosis \\
\hline Rodrigues et al. [97] & 2017 & Rats & Bilateral I/R injury & $\begin{array}{l}\uparrow \text { Renal function; } \downarrow \text { FENa; } \downarrow \text { urinary concentrating defect; } \downarrow \text { renal damage } \\
\text { score; } \downarrow \text { senescence }\end{array}$ \\
\hline
\end{tabular}

$I / R$ ischemia/reperfusion, $a$-SMA a-smooth muscle actin, MMP2 matrix metalloproteinase 2, FSP-1 fibroblast specific protein-1, PAI-1 plasminogen activator inhibitor-1 
problem is to remove the contralateral kidney on the basis of unilateral I/R injury, namely, unilateral I/R injury with contralateral nephrectomy injury [87].

The first study evaluating the long-term effects of MSC therapy on the AKI-CKD transition in this model was conducted by Gatti et al. in 2011. The administration of microvesicles (MVs) derived from MSCs immediately after right nephrectomy with left renal pedicle clamping significantly mitigated long-term kidney injury 6 months later. Proteinuria and fibrotic renal tissues in MV-treated rats were also both significantly lower than in the control group [88]. Similarly, the results originated from studies by Zou et al. and $\mathrm{Du}$ et al. indicated that a single injection of Wharton's jelly-derived MSCs (WJ-MSCs) after unilateral I/R injury with contralateral nephrectomy could reverse renal fibrosis and protect against CKD $[89,90]$. To closely examine the protective mechanism of MSC therapy, Alfarano et al. evaluated the development of CKD in their model of the AKICKD transition. Transplantation with bone marrowderived MSCs (BM-MSCs) improved renal function and modified renal remodeling in parallel with decreased accumulation of $\alpha$-SMA and metalloproteinase 2 (MMP2). Additionally, the authors further demonstrated that these beneficial mechanisms of MSC therapy might not account for its immunomodulatory or anti-inflammatory properties because of the usage of the immunosuppressive drug cyclosporine in their study, which indicated a direct activity of MSCs in the inhibition of fibrosis in renal tubular cells [91]. The stromal vascular fraction (SVF) was considered to be a rich source of MSC and also presented promising potential in delaying the AKICKD transition [92]. Zhou et al. attempted to engraft SVF into the kidneys of rats suffering from unilateral $I / R$ injury with contralateral nephrectomy. Six months later, declines in renal function and progression of fibrosis were both delayed by the administration of SVF, which strongly inhibited microvascular rarefaction [93].

\section{Application of MSCs in the bilateral I/R injury-induced AKI-CKD transition model}

Compared with the unilateral $I / R$ injury model and the unilateral $I / R$ injury with contralateral nephrectomy model, the bilateral I/R injury model is a more accurate model to study the AKI-CKD transition in humans. The bilateral I/R injury model is induced by the block of renal vessels in both kidneys, which may make the renal hemodynamic changes more relevant to human pathophysiological situations. Similar with the results obtained in the unilateral I/R injury with contralateral nephrectomy model, improvement of renal function and abrogation of renal fibrosis were observed in rats that received MSCs after suffering from bilateral I/R injury. Profibrotic genes, such as TGF- $\beta$ and tissue inhibitor of matrix metalloprotease-1 (TIMP-1), were also expressed in these kidneys at significantly lower levels, suggesting the anti-fibrotic effects of MSCs. However, after the effective knockdown of VEGF by siRNA in engrafted MSCs, all of the beneficial effects disappeared, indicating the importance of the VEGF pathway against a maladaptive repair in renal $\mathrm{I} / \mathrm{R}$ injury [94]. In addition to VEGF, HGF was another key factor in mediating the protective effects of MSCs against the AKI-CKD transition. A previous study reported that HGF could efficiently block the TGF- $\beta 1$ / Smad signaling [95]. To verify this hypothesis, a rat model of unilateral I/R injury was established. After 2 days, a single injection of WJ-MSCs was performed, and the ratio of HGF/TGF- $\beta 1$ was measured. Six weeks later, although without a change in the serum creatinine value, cell treatment significantly mitigated renal fibrosis triggered by the unilateral $I / R$ injury. In terms of mechanism, an upregulated ratio of HGF/TGF- $\beta 1$ was unambiguously observed after MSC transplantation. Via these experiments, the authors confirmed that MSC therapy might exert an important effect on the balance between HGF and TGF- $\beta 1$ during fibrogenesis [96]. Renal aging and Klotho are important factors in mediating the development of CKD. To evaluate their role in mediating the therapeutic effects of MSCs during the AKI repair process, Rodrigues et al. intraperitoneally injected MSCs into unilateral I/R injury-induced AKI rats. At day 49, senescence-related proteins and Klotho expression were both ameliorated in rats submitted to MSC therapy, which further helped to reverse renal dysfunction, urinary concentrating defect, and renal damage [97].

\section{Attempts with MSC therapy in clinical settings and challenges met in translation}

Above, we discussed the success of MSC therapy in preclinical models. What about the role of MSCs in clinical settings? Unfortunately, at this point, a clinical study assessing the role of MSC-based therapy in the field of the AKI-CKD transition is still lacking. However, as we know, maladaptive repair after AKI is a major risk factor for the development of the AKI-CKD transition. Here, we list the currently available clinical trials using MSC applications in AKI (Table 2). NCT00733876 was the first clinical trial assessing the safety and therapeutic effects of MSC transplantation in AKI patients. Allogeneic MSCs were prophylactically injected into 16 patients who were at high risk of developing AKI after undergoing on-pump cardiac surgery [98]. The administration was safe, and the outcome was positive, which encouraged the team to conduct a larger number, phase II, randomized, double-blind, multicenter trial in 2017 (NCT01602328) [99]. This trial included a total of 156 adult subjects who developed AKI after cardiac surgery. However, MSC treatment did not aid in renal recovery, 
Table 2 Available clinical trials of the application of MSCs in AKI

\begin{tabular}{|c|c|c|c|c|c|c|c|}
\hline ClinicalTrials.gov identifier & Year & Aim & MSC type & Enrollment & Phase & Status & Outcomes \\
\hline NCT00733876 & 2008 & $\begin{array}{l}\text { For the prevention of developing } \\
\text { AKI after cardiac surgery }\end{array}$ & Allogeneic & 16 & Phase I & Completed & $\begin{array}{l}\text { Safe and } \\
\text { effective }\end{array}$ \\
\hline NCT01602328 & 2017 & $\begin{array}{l}\text { For the treatment of AKI after } \\
\text { cardiac surgery }\end{array}$ & Allogeneic & 156 & Phase II & Terminated & $\begin{array}{l}\text { Safe but not } \\
\text { effective }\end{array}$ \\
\hline NCT01275612 & 2018 & $\begin{array}{l}\text { For the treatment of cisplatin- } \\
\text { induced AKI in cancer patients }\end{array}$ & Allogeneic & NM & Phase I & Withdrawn & NM \\
\hline NCT03015623 & 2018 & $\begin{array}{l}\text { For the treatment of AKI regardless } \\
\text { of the underlying reasons }\end{array}$ & $\begin{array}{l}\text { Allogeneic MSCs combined } \\
\text { with a biologic device }\end{array}$ & 24 & Phase I & Recruiting & $\begin{array}{l}\text { Date not } \\
\text { published }\end{array}$ \\
\hline
\end{tabular}

MSCs mesenchymal stem cells, $A K I$ acute kidney injury, NM not mentioned

and AKI duration, dialysis prevalence, and 30-day allcause mortality were comparable between the two groups. A highly anticipated study (NCT01275612) aimed at evaluating the feasibility and safety of the use of MSCs in the treatment of cisplatin-induced AKI was withdrawn recently. Based on these contradictory results, a third ongoing trial (NCT03015623) is currently recruiting. More research is needed to answer the question of whether MSC-based therapy can be effective in reducing the AKI-CKD transition in humans.

Why are promising preclinical results rarely verified in clinical settings? In our opinion, the restricted cell function after transplantation is a major factor in the poor translation outcomes. As we discussed in a previous article, the main reasons for the limited clinical efficacy were the low engraftment, the poor survival rate, and the impaired paracrine capacity of injected cells in vivo [100]. The majority of grafted MSCs might be trapped in the lungs, liver, and spleen. For those cells engrafted into the target tissues, the harsh microenvironment in vivo due to the activation of anoikis, ischemia, inflammation, and ROS production still would induce apoptosis in over 90\% of transplanted MSCs in 1 week [101]. Impaired MSC potency/biological activity is also common in vivo. Different preconditioning strategies have been designed to increase MSC engraftment, survival, and paracrine capacity in vivo and have presented wonderful results [102]. These facts have provided an explanation for the poor therapeutic effects in clinical trials.

In addition to the abovementioned difficulties during clinical translation, some failures were also met in animal models (Table 3). In a feline model of unilateral I/R injury-induced AKI, Rosselli et al. demonstrated that renal function, urine protein creatinine ratio $(\mathrm{UP} / \mathrm{C})$, SMA, and histopathologic scoring were all comparable in cats receiving MSC treatment and the control group [103]. Similarly, the results from a study by Fang et al. also indicated that hematopoietic lineage marrow cells (HLMCs) but not MSCs appeared to influence the course of $\mathrm{HgCl}_{2}$-induced AKI in mice [104]. Along with these negative results, the adverse effects deserve more attention. The renin-angiotensin system (RAS) has been reported to be involved in several key steps of bone marrow cell maturation processes [105]. To evaluate whether pathological RAS activation will have an impact on the therapeutic functionality of BM-MSCs, Kankuri et al. transplanted BM-MSCs derived from rats with increased RAS activation into rats suffering from bilateral I/R injuryinduced AKI. Unexpectedly, a dramatic deterioration of renal function was observed in the treatment group. Alterations in the host's RAS system induced a proinflammatory phenotype in BM-MSCs, which might compromise their therapeutic effects [106]. Meanwhile, although not conducted in an AKI setting, Kim et al. reported a case of a rapid deterioration of renal function after MSC treatment [107]. These lines of evidence highlighted the ineffectiveness and potential nephrotoxicity of MSCbased therapy; thus, it should be examined more closely before its translation into clinical applications.

Based on the lines of evidence mentioned above, there is still a long way to go before considering MSCs as a realistic clinical tool for the AKI-CKD transition. The challenges that need to be addressed here may also be of relevance for other approaches that utilize cell-based strategies in many other cases. First, a standard MSC regimen is needed. What kind of MSCs will serve as the best tissue source? BM-MSCs, umbilical cord-derived MSCs (UC-MSCs), A-MSCs, or induced pluripotent stem cell-derived MSCs (iPSC-MSCs)? Autologous or allogeneic? What is the best delivery route for transplantation? Intravenous, intra-arterial, intraperitoneal, or intrarenal injection? What is the best dosage and timing for AKI-CKD transition therapy? These discordances may induce apparent discrepancies in reports in the literature. Second, rigorous criteria for cell extraction, storage, and characterization are still lacking; as a result, MSCs are highly heterogeneous cells. Last but not least, safety expectations, including tumorigenicity and immunological compatibility, should be considered. Although there has not yet been a report of de novo tumor formation or significant immunogenicity in vivo following MSC injection in humans, these issues should always be kept in mind. Additional studies are warranted to address these issues. 
Table 3 Failed attempts of MSC treatment in animal models

\begin{tabular}{lllll}
\hline Reference & Year & Sample & Model & Renal outcomes \\
\hline Rosselli et al. [103] & 2015 & Cats & Unilateral I/R injury & No changes in renal function, UP/C, SMA, and histopathologic scoring \\
Fang et al. [104] & 2008 & Mice & $\mathrm{HgCl}_{2}$ & No contribution to renal tubular cells regeneration \\
Kankuri et al. [106] & 2015 & Rats & Bilateral I/R injury & Exacerbation of renal function \\
\hline
\end{tabular}

MSCs mesenchymal stem cells, $I / R$ ischemia-reperfusion, UP/C urine protein: creatinine, SMA smooth muscle actin

\section{Conclusion and future perspectives}

By summarizing the currently available research, we would like to conclude that therapeutic strategies based on MSCs are no doubt promising for the management of the AKI-CKD transition. However, before the clinical application of MSCs, some issues remain to be solved in future research studies.

First, although most studies support the beneficial role of MSCs in the management of the AKI-CKD transition, the heterogeneity of cell populations makes MSC transplantation a fundamentally different strategy from most pharmacologic interventions. Different MSC subtype characteristics, differences in protocols used to obtain the cells, the lack of a standardized MSC preparation method, and other inconsistencies might help us to explain why there are still some contradictory results in the literature. The complexity of different therapeutic regimens could also contribute to the controversial results. Conducting coordinated studies comparing the effects of distinct subpopulations of MSCs in therapeutic therapy regimens may give us a clearer insight into these questions.

The second issue regards the model of the AKI-CKD transition. I/R injury models are still the best developed and most widely applied animal models in the research field of the AKI-CKD transition. According to different surgical procedures, these models can be classified into bilateral I/R injury, unilateral I/R injury, and unilateral I/ $\mathrm{R}$ injury with contralateral nephrectomy. In addition to $\mathrm{I} / \mathrm{R}$ injury models, repeated low-dose nephrotoxicity drug stimulation may also cause long-term side effects in the kidneys and lead to an AKI-CKD transition. The advantages and pitfalls of these models were mentioned above and are summarized in Fig. 2. While I/R injury models provide the consistency, reliability, and capacity of long-term observation, nephrotoxic models are also a good supplement to I/R injury models for understanding the different causes and mechanisms of the AKI-CKD transition, especially in cancer patients who intermittently receive cisplatin therapy.

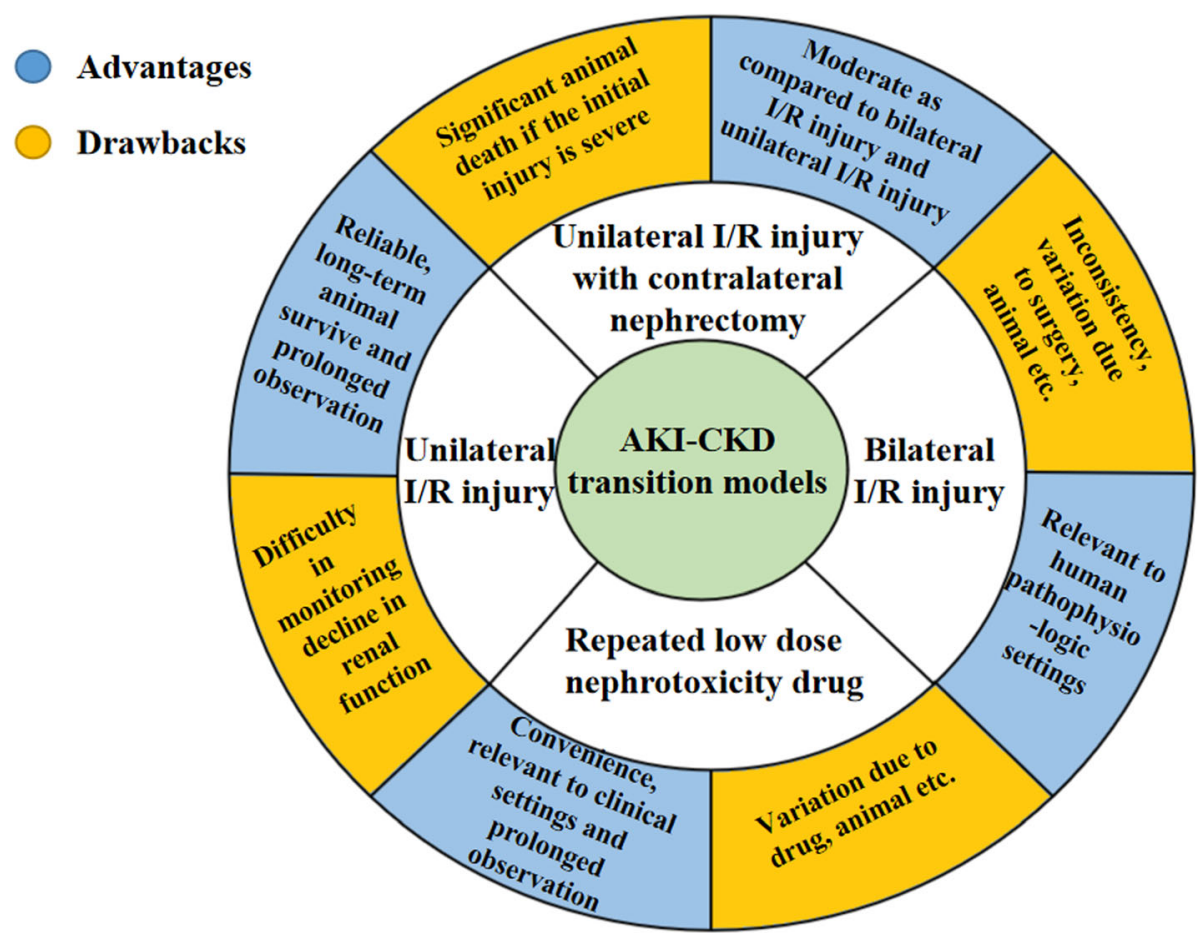

Fig. 2 Advantages and pitfalls of different animal models in AKI-CKD transition research 
Another important issue is the definition of the AKICKD transition. A consensus about the definition of AKI recovery is still lacking. Serum creatinine and estimated glomerular filtration rate (eGFR) are used as traditional biomarkers for the judgment of the existence of renal injury. However, due to the low sensitivity of serum creatinine and eGFR in diagnosing kidney disease, these markers are insufficient for precisely reflecting the pathophysiological processes during the AKI-CKD transition. To overcome this shortcoming, the 13th Acute Dialysis Quality Initiative Meeting described adaptive repair as a phenomenon without long-term sequelae in renal function and structure over a period of three months after AKI [9]. However, this definition largely depends on renal biopsy, which may limit its clinical application due to its invasive property. In our opinion, establishment of a standard definition for the AKI-CKD transition by application of other novel biomarkers, such as neutrophil gelatinase-associated lipocalin (NGAL), kidney injury molecule-1 (KIM-1), nephrin, podocalyxin, IL-18, and other biomarkers, could help to provide early diagnosis and allow for a timely intervention for these patients, which is urgently needed.

Finally, we need to establish a post-AKI monitoring system. Despite developing in the field of pharmaceutical research, the best way to reduce AKI-CKD continuum is still prevention. However, given that the severity, duration, and frequency of repeat episodes of AKI are critical points of its poor outcome, the clinical follow-up rate of AKI survivors is still extremely low at present $[108,109]$. While advancing potential therapies, in the meantime, there is an urgent need to define those patients who are most at risk of developing CKD after an episode of AKI. Exploring novel protocols, including essential components and precise timing for monitoring patients who suffer from AKI, is of great importance.

In conclusion, although there is still no reliable therapeutic approach for the treatment of the AKI-CKD transition at present, MSCs have the potential to block AKICKD transition, at least partially. Randomized controlled clinical trials should be initiated, and we call for more research in this area.

\footnotetext{
Abbreviations

AKl: Acute kidney injury; A-MSCs: Adipose-derived MSCs; ATP: Adenosine triphosphate; BMMCs: Bone marrow mononuclear cells; BM-MSCs: Bone marrow-derived MSCs; CKD: Chronic kidney disease; EGFR: Epidermal growth factor receptor; eGFR: Estimated glomerular filtration rate; EVs: Extracellular vesicles; FGF: Fibroblast growth factor; FSP-1: Fibroblast-specific protein-1; HGF: Hepatocyte growth factor; HIF: Hypoxia-inducible factor; HLMCs: Hematopoietic lineage marrow cells; I/R: Ischemia-reperfusion; IGF1: Insulin-like growth factor type 1; iPSC-MSCs: Induced pluripotent stem cellderived MSCs; JNK: c-jun NH2-terminal kinase; KIM-1: Kidney injury molecule1; MMP2: Metalloproteinase 2; MSCs: Mesenchymal stem cells; MVs: Microvesicles; NGAL: Neutrophil gelatinase-associated lipocalin; Nrf2: Nuclear factor erythroid-2-related factor 2; PAI-1: Plasminogen activator inhibitor-1; PDGFR- $\beta$ : Platelet-derived growth factor receptor- $\beta$; PTEN: Phosphatase and tensin homolog; RAS: Renin-angiotensin system;
}

RASI: Renin-angiotensin system inhibitor; ROS: Reactive oxygen species; SVF: Stromal vascular fraction; TECs: Tubular epithelial cells; TGF$\beta$ : Transforming growth factor- $\beta$; TIMP-1: Tissue inhibitor of matrix metalloprotease-1; TLRs: Toll-like receptors; TNF-a: Tumor necrosis factor-a; UC-MSCs: Umbilical cord-derived MSCs; UP/C: Urine protein creatinine ratio VEGF: Vascular endothelial growth factor; WJ-MSCs: Wharton's jelly-derived MSCs; a-SMA: a-Smooth muscle actin

\section{Acknowledgements}

The authors would like to thank the laboratory members for their contributions and funding support from the sources indicated.

\section{Authors' contributions}

LZ and JC contributed to the conception of this manuscript. LZ and FH were responsible for the literature review. LZ, FH, and JW drafted and revised the manuscript. All authors read and approved the final manuscript.

\section{Funding}

This work is supported by the National Natural Science Foundation of China (No. 81700553).

\section{Availability of data and materials}

Not applicable

Ethics approval and consent to participate

Not applicable

\section{Consent for publication}

Not applicable

\section{Competing interests}

The authors declare that they have no competing interests.

Received: 28 July 2019 Revised: 3 November 2019

Accepted: 22 November 2019 Published online: 16 December 2019

\section{References}

1. Fraser S, Roderick PJ. Kidney disease in the Global Burden of Disease Study 2017. Nat Rev Nephrol. 2019;15(4):193-4.

2. Saran R, Li Y, Robinson B, et al. US Renal Data System 2015 Annual Data Report: Epidemiology of Kidney Disease in the United States. Am J Kidney Dis. 2016:67(3 Suppl 1):Svii, S1-305.

3. Honeycutt AA, Segel JE, Zhuo X, Hoerger TJ, Imai K, Williams D. Medical costs of CKD in the Medicare population. J Am Soc Nephrol. 2013;24(9):1478-83.

4. Chawla LS, Eggers PW, Star RA, Kimmel PL. Acute kidney injury and chronic kidney disease as interconnected syndromes. N Engl J Med. 2014;371(1):58-66.

5. Feng G, Zhang J, Li Y, et al. IGF-1 C domain-modified hydrogel enhances cell therapy for AKI. J Am Soc Nephrol. 2016;27(8):2357-69.

6. Strausser SA, Nakano D, Souma T. Acute kidney injury to chronic kidney disease transition: insufficient cellular stress response. Curr Opin Nephrol Hypertens. 2018;27(4):314-22.

7. LeBleu VS, Taduri G, O'Connell J, et al. Origin and function of myofibroblasts in kidney fibrosis. Nat Med. 2013;19(8):1047-53.

8. Chawla LS, Amdur RL, Amodeo S, Kimmel PL, Palant CE. The severity of acute kidney injury predicts progression to chronic kidney disease. Kidney Int. 2011;79(12):1361-9.

9. Basile DP, Bonventre JV, Mehta R, et al. Progression after AKl: understanding maladaptive repair processes to predict and identify therapeutic treatments. J Am Soc Nephrol. 2016;27(3):687-97.

10. Kapitsinou PP, Jaffe J, Michael M, et al. Preischemic targeting of HIF prolyl hydroxylation inhibits fibrosis associated with acute kidney injury. Am J Physiol Renal Physiol. 2012;302(9):F1172-9.

11. Leonard EC, Friedrich JL, Basile DP. VEGF-121 preserves renal microvessel structure and ameliorates secondary renal disease following acute kidney injury. Am J Physiol Renal Physiol. 2008;295(6):F1648-57.

12. Haase VH. Hypoxia-inducible factors in the kidney. Am J Physiol Renal Physiol. 2006;291(2):F271-81.

13. Liu M, Reddy NM, Higbee EM, et al. The Nrf2 triterpenoid activator, CDDOimidazolide, protects kidneys from ischemia-reperfusion injury in mice. Kidney Int. 2014;85(1):134-41. 
14. Barrera-Chimal J, Pérez-Villalva R, Rodríguez-Romo R, et al. Spironolactone prevents chronic kidney disease caused by ischemic acute kidney injury. Kidney Int. 2013;83(1):93-103.

15. Chou YH, Huang TM, Pan SY, et al. Renin-angiotensin system inhibitor is associated with lower risk of ensuing chronic kidney disease after functional recovery from acute kidney injury. Sci Rep. 2017;7:46518.

16. Charbord P. Bone marrow mesenchymal stem cells: historical overview and concepts. Hum Gene Ther. 2010;21(9):1045-56.

17. Prodromidi El, Poulsom R, Jeffery $\mathrm{R}$, et al. Bone marrow-derived cells contribute to podocyte regeneration and amelioration of renal disease in a mouse model of Alport syndrome. Stem Cells. 2006;24(11):2448-55.

18. Zhao DC, Lei JX, Chen R, et al. Bone marrow-derived mesenchymal stem cells protect against experimental liver fibrosis in rats. World J Gastroenterol. 2005;11(22):3431-40.

19. Ortiz LA, Gambelli F, McBride C, et al. Mesenchymal stem cell engraftment in lung is enhanced in response to bleomycin exposure and ameliorates its fibrotic effects. Proc Natl Acad Sci U S A. 2003;100(14):8407-11.

20. Liu KD, Brakeman PR. Renal repair and recovery. Crit Care Med. 2008;36(4 Suppl):S187-92.

21. Tsubokawa T, Yagi K, Nakanishi C, et al. Impact of anti-apoptotic and antioxidative effects of bone marrow mesenchymal stem cells with transient overexpression of heme oxygenase-1 on myocardial ischemia. Am J Physiol Heart Circ Physiol. 2010;298(5):H1320-9.

22. Hoogduijn MJ, Popp F, Verbeek R, et al. The immunomodulatory properties of mesenchymal stem cells and their use for immunotherapy. Int Immunopharmacol. 2010;10(12):1496-500.

23. Kim HK, Lee SG, Lee SW, et al. A subset of paracrine factors as efficient biomarkers for predicting vascular regenerative efficacy of mesenchymal stromal/stem cells. Stem Cells. 2019;37(1):77-88.

24. Yun CW, Lee SH. Potential and therapeutic efficacy of cell-based therapy using mesenchymal stem cells for acute/chronic kidney disease. Int J Mol Sci. 2019;20(7):1619.

25. Aghajani Nargesi A, Lerman LO, Eirin A. Mesenchymal stem cell-derived extracellular vesicles for kidney repair: current status and looming challenges. Stem Cell Res Ther. 2017;8(1):273.

26. Venkatachalam MA, Weinberg JM, Kriz W, Bidani AK. Failed tubule recovery, AKI-CKD transition, and kidney disease progression. J Am Soc Nephrol. 2015; 26(8):1765-76.

27. Pannu N. Bidirectional relationships between acute kidney injury and chronic kidney disease. Curr Opin Nephrol Hypertens. 2013;22(3):351-6.

28. Humphreys BD, Czerniak S, DiRocco DP, Hasnain W, Cheema R, Bonventre $J V$. Repair of injured proximal tubule does not involve specialized progenitors. Proc Natl Acad Sci U S A. 2011;108(22):9226-31.

29. Humphreys BD, Valerius MT, Kobayashi A, et al. Intrinsic epithelial cells repair the kidney after injury. Cell Stem Cell. 2008;2(3):284-91.

30. Bonventre JV. Dedifferentiation and proliferation of surviving epithelial cells in acute renal failure. J Am Soc Nephrol. 2003;14(Suppl 1):S55-61.

31. Bonventre JV, Yang L. Cellular pathophysiology of ischemic acute kidney injury. J Clin Invest. 2011;121(11):4210-21.

32. Lin SL, Chang FC, Schrimpf C, et al. Targeting endothelium-pericyte cross talk by inhibiting VEGF receptor signaling attenuates kidney microvascular rarefaction and fibrosis. Am J Pathol. 2011;178(2):911-23.

33. Hörbelt M, Lee SY, Mang HE, et al. Acute and chronic microvascular alterations in a mouse model of ischemic acute kidney injury. Am J Physiol Renal Physiol. 2007;293(3):F688-95.

34. Kramann R, Wongboonsin J, Chang-Panesso M, Machado FG, Humphreys BD. Gli1+ pericyte loss induces capillary rarefaction and proximal tubular injury. J Am Soc Nephrol. 2017;28(3):776-84.

35. Yang L, Besschetnova TY, Brooks CR, Shah JV, Bonventre JV. Epithelial cell cycle arrest in G2/M mediates kidney fibrosis after injury. Nat Med. 2010; 16(5):535-43 1p following 143.

36. Ferenbach DA, Bonventre JV. Mechanisms of maladaptive repair after AKI leading to accelerated kidney ageing and CKD. Nat Rev Nephrol. 2015;11(5):264-76.

37. Humphreys $\mathrm{BD}$, Lin $\mathrm{SL}$, Kobayashi $\mathrm{A}$, et al. Fate tracing reveals the pericyte and not epithelial origin of myofibroblasts in kidney fibrosis. Am J Pathol. 2010;176(1):85-97.

38. Basile DP, Friedrich $J$, Spahic J, et al. Impaired endothelial proliferation and mesenchymal transition contribute to vascular rarefaction following acute kidney injury. Am J Physiol Renal Physiol. 2011;300(3):F721-33.

39. Maringer $K$, Sims-Lucas $S$. The multifaceted role of the renal microvasculature during acute kidney injury. Pediatr Nephrol. 2016;31(8):1231-40.
40. Dimke H, Sparks MA, Thomson BR, Frische S, Coffman TM, Quaggin SE. Tubulovascular cross-talk by vascular endothelial growth factor a maintains peritubular microvasculature in kidney. J Am Soc Nephrol. 2015;26(5):1027-38.

41. Bábíčková J, Klinkhammer BM, Buhl EM, et al. Regardless of etiology, progressive renal disease causes ultrastructural and functional alterations of peritubular capillaries. Kidney Int. 2017;91(1):70-85.

42. Basile DP. The endothelial cell in ischemic acute kidney injury: implications for acute and chronic function. Kidney Int. 2007;72(2):151-6.

43. Kramann R, Tanaka M, Humphreys BD. Fluorescence microangiography for quantitative assessment of peritubular capillary changes after AKI in mice. J Am Soc Nephrol. 2014;25(9):1924-31.

44. Zhao L, Hu C, Zhang P, Jiang H, Chen J. Mesenchymal stem cell therapy targeting mitochondrial dysfunction in acute kidney injury. J Transl Med. 2019;17(1):142.

45. Lan R, Geng H, Singha PK, et al. Mitochondrial pathology and glycolytic shift during proximal tubule atrophy after ischemic AKI. J Am Soc Nephrol. 2016; 27(11):3356-67.

46. Perry HM, Huang L, Wilson RJ, et al. Dynamin-related protein 1 deficiency promotes recovery from AKI. J Am Soc Nephrol. 2018;29(1):194-206.

47. Stallons $\sqcup$, Whitaker RM, Schnellmann RG. Suppressed mitochondrial biogenesis in folic acid-induced acute kidney injury and early fibrosis. Toxicol Lett. 2014;224(3):326-32.

48. Szeto HH, Liu S, Soong Y, et al. Mitochondria protection after acute ischemia prevents prolonged upregulation of IL-1 $\beta$ and IL-18 and arrests CKD. J Am Soc Nephrol. 2017;28(5):1437-49.

49. Kang HM, Ahn SH, Choi P, et al. Defective fatty acid oxidation in renal tubular epithelial cells has a key role in kidney fibrosis development. Nat Med. 2015;21(1):37-46

50. Novitskaya T, McDermott L, Zhang KX, et al. A PTBA small molecule enhances recovery and reduces postinjury fibrosis after aristolochic acidinduced kidney injury. Am J Physiol Renal Physiol. 2014;306(5):F496-504.

51. Cianciolo Cosentino C, Skrypnyk NI, Brilli LL, et al. Histone deacetylase inhibitor enhances recovery after AKI. J Am Soc Nephrol. 2013;24(6):943-53.

52. Cedar H, Bergman Y. Linking DNA methylation and histone modification: patterns and paradigms. Nat Rev Genet. 2009;10(5):295-304.

53. Tampe B, Steinle U, Tampe D, et al. Low-dose hydralazine prevents fibrosis in a murine model of acute kidney injury-to-chronic kidney disease progression. Kidney Int. 2017;91(1):157-76.

54. Leemans JC, Kors L, Anders HJ, Florquin S. Pattern recognition receptors and the inflammasome in kidney disease. Nat Rev Nephrol. 2014;10(7):398-414.

55. Liu D, Wen Y, Tang TT, et al. Megalin/cubulin-lysosome-mediated albumin reabsorption is involved in the tubular cell activation of NLRP3 inflammasome and tubulointerstitial inflammation. J Biol Chem. 2015; 290(29):18018-28.

56. Ding LH, Liu D, Xu M, et al. TLR2-MyD88-NF-KB pathway is involved in tubulointerstitial inflammation caused by proteinuria. Int J Biochem Cell Biol. 2015:69:114-20.

57. Wang S, Diao H, Guan Q, et al. Decreased renal ischemia-reperfusion injury by IL-16 inactivation. Kidney Int. 2008;73(3):318-26.

58. Kang DH, Joly AH, Oh SW, et al. Impaired angiogenesis in the remnant kidney model: I. potential role of vascular endothelial growth factor and thrombospondin-1. J Am Soc Nephrol. 2001;12(7):1434-47.

59. Anders HJ. Of inflammasomes and alarmins: IL-1 $\beta$ and IL-1a in kidney disease. J Am Soc Nephrol. 2016;27(9):2564-75.

60. Weiler M, Kachko L, Chaimovitz C, Van Kooten C, Douvdevani A. CD40 ligation enhances IL-15 production by tubular epithelial cells. J Am Soc Nephrol. 2001;12(1):80-7.

61. Yard BA, Daha MR, Kooymans-Couthino M, et al. IL-1 alpha stimulated TNF alpha production by cultured human proximal tubular epithelial cells. Kidney Int. 1992;42(2):383-9.

62. Wang Y, Chang J, Yao B, et al. Proximal tubule-derived colony stimulating factor-1 mediates polarization of renal macrophages and dendritic cells, and recovery in acute kidney injury. Kidney Int. 2015;88(6):1274-82.

63. Baek JH, Zeng R, Weinmann-Menke J, et al. IL-34 mediates acute kidney injury and worsens subsequent chronic kidney disease. J Clin Invest. 2015;125(8):3198-214.

64. Zhou D, Li Y, Lin L, Zhou L, Igarashi P, Liu Y. Tubule-specific ablation of endogenous $\beta$-catenin aggravates acute kidney injury in mice. Kidney Int. 2012;82(5):537-47.

65. Terada $Y$, Tanaka H, Okado $T$, et al. Expression and function of the developmental gene Wnt-4 during experimental acute renal failure in rats. J Am Soc Nephrol. 2003;14(5):1223-33. 
66. Xiao L, Zhou D, Tan RJ, et al. Sustained activation of Wnt/B-catenin signaling drives AKI to CKD progression. J Am Soc Nephrol. 2016;27(6):1727-40.

67. Cao Y, Hu J, Sui J, Jiang L, Cong Y, Ren G. Quercetin is able to alleviate TGF$\beta$-induced fibrosis in renal tubular epithelial cells by suppressing miR-21. Exp Ther Med. 2018;16(3):2442-8.

68. Godwin JG, Ge X, Stephan K, Jurisch A, Tullius SG, lacomini J. Identification of a microRNA signature of renal ischemia reperfusion injury. Proc Natl Acad Sci U S A. 2010;107(32):14339-44.

69. Tang J, Liu N, Tolbert E, et al. Sustained activation of EGFR triggers renal fibrogenesis after acute kidney injury. Am J Pathol. 2013;183(1):160-72.

70. Wu CF, Chiang WC, Lai CF, et al. Transforming growth factor $\beta-1$ stimulates profibrotic epithelial signaling to activate pericyte-myofibroblast transition in obstructive kidney fibrosis. Am J Pathol. 2013;182(1):118-31.

71. Armulik A, Abramsson A, Betsholtz C. Endothelial/pericyte interactions. Circ Res. 2005;97(6):512-23.

72. Duffield JS. Cellular and molecular mechanisms in kidney fibrosis. J Clin Invest. 2014;124(6):2299-306.

73. Fligny C, Duffield JS. Activation of pericytes: recent insights into kidney fibrosis and microvascular rarefaction. Curr Opin Rheumatol. 2013;25(1):78-86.

74. Liu BC, Tang $\Pi$, Lv LL, Lan HY. Renal tubule injury: a driving force toward chronic kidney disease. Kidney Int. 2018;93(3):568-79.

75. Venkatachalam MA, Griffin KA, Lan R, Geng H, Saikumar P, Bidani AK. Acute kidney injury: a springboard for progression in chronic kidney disease. Am J Physiol Renal Physiol. 2010;298(5):F1078-94.

76. Qian $\mathrm{H}$, Yang $\mathrm{H}$, Xu W, et al. Bone marrow mesenchymal stem cells ameliorate rat acute renal failure by differentiation into renal tubular epithelial-like cells. Int J Mol Med. 2008;22(3):325-32.

77. Peired A, Sisti A, Romagnani P. Mesenchymal stem cell-based therapy for kidney disease: a review of clinical evidence. Stem Cells Int. 2016;2016:4798639.

78. He A, Jiang Y, Gui C, Sun Y, Li J, Wang JA. The antiapoptotic effect of mesenchymal stem cell transplantation on ischemic myocardium is enhanced by anoxic preconditioning. Can J Cardiol. 2009;25(6):353-8.

79. Valle-Prieto A, Conget PA. Human mesenchymal stem cells efficiently manage oxidative stress. Stem Cells Dev. 2010;19(12):1885-93.

80. Roudkenar MH, Halabian R, Tehrani HA, et al. Lipocalin 2 enhances mesenchymal stem cell-based cell therapy in acute kidney injury rat model. Cytotechnology. 2018;70(1):103-17.

81. Nawaz M, Fatima F, Vallabhaneni KC, et al. Extracellular vesicles: evolving factors in stem cell biology. Stem Cells Int. 2016;2016:1073140.

82. Zhao L, Hu C, Zhang P, Jiang H, Chen J. Genetic communication by extracellular vesicles is an important mechanism underlying stem cell-based therapy-mediated protection against acute kidney injury. Stem Cell Res Ther. 2019;10(1):119.

83. Zager RA, Johnson AC, Becker K. Acute unilateral ischemic renal injury induces progressive renal inflammation, lipid accumulation, histone modification, and "end-stage" kidney disease. Am J Physiol Renal Physiol. 2011;301(6):F1334-45.

84. Zhu F, Chong Lee Shin O, Pei G, et al. Adipose-derived mesenchymal stem cells employed exosomes to attenuate AKI-CKD transition through tubular epithelial cell dependent Sox9 activation. Oncotarget. 2017;8(41):70707-26

85. Masoud MS, Anwar SS, Afzal MZ, Mehmood A, Khan SN, Riazuddin S. Preconditioned mesenchymal stem cells ameliorate renal ischemic injury in rats by augmented survival and engraftment. J Transl Med. 2012;10:243.

86. Semedo P, Donizetti-Oliveira C, Burgos-Silva M, et al. Bone marrow mononuclear cells attenuate fibrosis development after severe acute kidney injury. Lab Investig. 2010;90(5):685-95.

87. Skrypnyk NI, Harris RC, de Caestecker MP. Ischemia-reperfusion model of acute kidney injury and post injury fibrosis in mice. J Vis Exp. 2013;(78): e50495.

88. Gatti S, Bruno S, Deregibus MC, et al. Microvesicles derived from human adult mesenchymal stem cells protect against ischaemia-reperfusion-induced acute and chronic kidney injury. Nephrol Dial Transplant. 2011;26(5):1474-83.

89. Zou X, Zhang G, Cheng Z, et al. Microvesicles derived from human Wharton's jelly mesenchymal stromal cells ameliorate renal ischemia-reperfusion injury in rats by suppressing CX3CL1. Stem Cell Res Ther. 2014:5(2):40.

90. Du T, Cheng J, Zhong L, et al. The alleviation of acute and chronic kidney injury by human Wharton's jelly-derived mesenchymal stromal cells triggered by ischemia-reperfusion injury via an endocrine mechanism. Cytotherapy. 2012;14(10):1215-27.

91. Alfarano C, Roubeix C, Chaaya R, et al. Intraparenchymal injection of bone marrow mesenchymal stem cells reduces kidney fibrosis after ischemia- reperfusion in cyclosporine-immunosuppressed rats. Cell Transplant. 2012; 21(9):2009-19.

92. Gentile P, Orlandi A, Scioli MG, Di Pasquali C, Bocchini I, Cervelli V. Concise review: adipose-derived stromal vascular fraction cells and platelet-rich plasma: basic and clinical implications for tissue engineering therapies in regenerative surgery. Stem Cells Transl Med. 2012;1(3):230-6.

93. Zhou L, Xu L, Shen J, et al. Preischemic administration of nonexpanded adipose stromal vascular fraction attenuates acute renal ischemia/ reperfusion injury and fibrosis. Stem Cells Transl Med. 2016;5(9):1277-88.

94. Tögel F, Cohen A, Zhang P, Yang Y, Hu Z, Westenfelder C. Autologous and allogeneic marrow stromal cells are safe and effective for the treatment of acute kidney injury. Stem Cells Dev. 2009;18(3):475-85.

95. Yang J, Dai C, Liu Y. A novel mechanism by which hepatocyte growth factor blocks tubular epithelial to mesenchymal transition. J Am Soc Nephrol. 2005;16(1):68-78.

96. Du T, Zou X, Cheng J, et al. Human Wharton's jelly-derived mesenchymal stromal cells reduce renal fibrosis through induction of native and foreign hepatocyte growth factor synthesis in injured tubular epithelial cells. Stem Cell Res Ther. 2013;4(3):59.

97. Rodrigues CE, Capcha JM, de Bragança AC, et al. Human umbilical cordderived mesenchymal stromal cells protect against premature renal senescence resulting from oxidative stress in rats with acute kidney injury. Stem Cell Res Ther. 2017:8(1):19.

98. Tögel FE, Westenfelder C. Kidney protection and regeneration following acute injury: progress through stem cell therapy. Am J Kidney Dis. 2012; 60(6):1012-22

99. Swaminathan M, Stafford-Smith M, Chertow GM, et al. Allogeneic mesenchymal stem cells for treatment of AKI after cardiac surgery. J Am Soc Nephrol. 2018;29(1):260-7.

100. Zhao L, Hu C, Zhang P, Jiang $H$, Chen J. Preconditioning strategies for improving the survival rate and paracrine ability of mesenchymal stem cells in acute kidney injury. J Cell Mol Med. 2019;23(2):720-30.

101. Zhang M, Methot D, Poppa V, Fujio Y, Walsh K, Murry CE. Cardiomyocyte grafting for cardiac repair: graft cell death and anti-death strategies. J Mol Cell Cardiol. 2001;33(5):907-21.

102. Zhao L, Hu C, Zhang P, Jiang H, Chen J. Novel preconditioning strategies for enhancing the migratory ability of mesenchymal stem cells in acute kidney injury. Stem Cell Res Ther. 2018;9(1):225.

103. Rosselli DD, Mumaw JL, Dickerson V, Brown CA, Brown SA, Schmiedt CW. Efficacy of allogeneic mesenchymal stem cell administration in a model of acute ischemic kidney injury in cats. Res Vet Sci. 2016;108:18-24.

104. Fang TC, Otto WR, Rao J, et al. Haematopoietic lineage-committed bone marrow cells, but not cloned cultured mesenchymal stem cells, contribute to regeneration of renal tubular epithelium after $\mathrm{HgCl}_{2}$-induced acute tubular injury. Cell Prolif. 2008;41(4):575-91.

105. Sinka L, Biasch K, Khazaal I, Péault B, Tavian M. Angiotensin-converting enzyme (CD143) specifies emerging lympho-hematopoietic progenitors in the human embryo. Blood. 2012;119(16):3712-23.

106. Kankuri E, Mervaala EE, Storvik M, et al. Exacerbation of acute kidney injury by bone marrow stromal cells from rats with persistent renin-angiotensin system activation. Clin Sci (Lond). 2015;128(11):735-47.

107. Kim JS, Lee JH, Kwon O, et al. Rapid deterioration of preexisting renal insufficiency after autologous mesenchymal stem cell therapy. Kidney Res Clin Pract. 2017;36(2):200-4.

108. Goldstein SL, Jaber BL, Faubel S, Chawla LS, Acute Kidney Injury Advisory Group of American Society of Nephrology. AKI transition of care: a potential opportunity to detect and prevent CKD. Clin J Am Soc Nephrol. 2013;8(3): 476-83.

109. Heung M, Steffick DE, Zivin K, et al. Acute kidney injury recovery pattern and subsequent risk of CKD: an analysis of veterans health administration data. Am J Kidney Dis. 2016;67(5):742-52.

\section{Publisher's Note}

Springer Nature remains neutral with regard to jurisdictional claims in published maps and institutional affiliations. 